\title{
Ist automatisiertes Fahren nachhaltig?
}

\author{
Entwicklungstendenzen und Forschungsüberlegungen \\ angesichts geringer Akzeptanz
}

Karsten Weber, Institut für Sozialforschung und Technikfolgenabschätzung, Ostbayerische Technische Hochschule Regensburg, Galgenbergstraße 24, 93053 Regensburg (Karsten.Weber@oth-regensburg.de), (1) orcid.org/0000-0001-8875-2386

Sonja Haug, Institut für Sozialforschung und Technikfolgenabschätzung, Ostbayerische Technische Hochschule Regensburg (Sonja.Haug@oth-regensburg.de)

Automatisiertes Fahren stößt derzeit noch auf große Skepsis. Eine disruptive Strategie bei der Einführung (voll-)automatisierten Fahrens könnte daher auf fehlende Akzeptanz treffen. Um dem zu entgehen, laufen evolutionäre Strategien darauf hinaus, durch die Entwicklung adaptiver Fahrassistenzsysteme Vertrautheit, Vertrauen und damit Akzeptanz bei den prospektiven NutzerInnen zu schaffen. Erste Ergebnisse einer Pilotstudie lassen jedoch Zweifel an der Nachhaltigkeit dieser Strategie aufkommen.

\section{Is automated driving sustainable?}

Development trends and research considerations in view of

low acceptance rates

Automated driving is currently viewed with great skepticism. A disruptive strategy for the introduction of (fully) automated driving could therefore be met with a lack of acceptance. To avoid this, evolutionary strategies aim to create familiarity, trust, and thus acceptance among the prospective users through the development of adaptive driver assistance systems. However, the preliminary results of a pilot study raise doubts about the sustainability of this strategy.

Keywords: driver assistance systems, automated driving, acceptance, sustainability

\section{Einleitung}

Verschiedene aktuelle Studien (z. B. Bertelsmann 2017; Ernst \& Young 2017; Deloitte 2017) weisen auf, dass vollautomatisiertes Fahren auf erhebliche Skepsis stößt: Bis zu zwei Drittel der in den Studien Befragten äußern Ablehnung in Hinblick auf vollautomatisierte Pkw, oft mit dem Hinweis auf die Unsicherheit der Technik. Die fehlende Akzeptanz erklärt sich vermutlich u. a. durch Berichte über Unfälle mit vermeintlich vollautomatisierten Pkw, doch könnte deren Wurzel auch in der Angst vor Kontrollverlust und grundsätzlichem Misstrauen gegenüber einer weitgehend unbekannten Technik liegen, mit der außerhalb von Testzentren kaum jemand umfassende Erfahrung hat. Akzeptanzmindernd könnte zudem sein, dass vollautomatisiertes Fahren es erschweren bis verunmöglichen würde, einem eigenen Fahrstil Ausdruck zu verleihen. Der Untersuchung dieser Vermutung ist der vorliegende Beitrag gewidmet.

Firmen, die bisher nicht als Automobilbauer aufgetreten sind - z. B. Uber, Apple oder Google -, scheinen einen disruptiven Ansatz zu verfolgen und möglichst viele Zwischenphasen zum vollautomatisierten Fahren überspringen zu wollen, um sofort mit führerlosen Fahrzeugen neue Geschäftsmodelle anbieten zu können. „Disruptiv“ bezieht sich dabei nicht auf die verbaute Fahrzeugtechnik (Sensoren, Aktoren, Steuerungsrechner etc.), sondern in erster Linie auf die Möglichkeit bzw. Unmöglichkeit auf das Fahrzeugverhalten einwirken zu können. Ein fahrerloser Pkw oder Lkw bedeutet einen radikalen Bruch mit rund 120 Jahren Automobilgeschichte und einer noch längeren Geschichte menschlicher Mobilität. Die Disruption findet also aus Perspektive der FahrzeugnutzerInnen und anderer VerkehrsteilnehmerInnen statt.

Andere Unternehmen, z. B. die großen deutschen Automobilhersteller, setzen auf eine evolutionäre Entwicklung mit einer schrittweisen und jeweils partiellen Übernahme von Steuerungsaufgaben durch Fahrassistenzsysteme, was den FahrzeugnutzerInnen ebenso wie anderen VerkehrsteilnehmerInnen die Chance
This is an article distributed under the terms of the Creative Commons Attribution License CCBY 4.0 (https://creativecommons.org/licenses/by/4.0/)

https://doi.org/10.14512/tatup.27.2.16

Submitted: 23. 02.2018. Peer reviewed. Accepted: 11. 05.2018 
einer langsamen Gewöhnung an die für sie neue Technik eröffnet. Als Konsequenz daraus würden noch über lange Zeit hinweg sowohl rein von Menschen gesteuerte, teilassistierte als auch (irgendwann) hoch- und vollautomatisierte Fahrzeuge gleichzeitig auf den Straßen unterwegs sein. Die gültige Rechtslage scheint diese Strategie zu bestätigen, denn vorerst wird die Haftung bei den FahrzeughalterInnen verbleiben - was aber den Wunsch nach Kontrolle über das Fahrzeug aufseiten der FahrerInnen verstärken und die Akzeptanz vollautomatisierten Fahrens verringern könnte.

Obwohl vermutet werden kann, dass bei der Wahl einer evolutionären Entwicklung nicht zuletzt Marktüberlegungen und damit ökonomische Faktoren eine wichtige Rolle spielen, kann sie auch dazu geeignet sein, Ängste aufseiten der Öffentlichkeit abzubauen sowie die Akzeptanz für Fahrassistenzsysteme und, auf längere Sicht, für automatisiertes Fahren der drei höchsten Stufen (hochautomatisiert, vollautomatisiert, fahrerlos) bei den prospektiven FahrerInnen zu erhöhen. Im Folgenden soll daher etwas ausführlicher auf diese evolutionäre Strategie eingegangen werden, da sie die Motivation der später beschriebenen Pilotstudie liefert. Mit dieser Studie sollten Grundlagen zur fahrerbasierten Parametrisierung von Fahrassistenzsystemen erarbeitet werden, doch die Ergebnisse lassen zusätzlich Schlüsse in Bezug auf die Frage zu, ob parametrisierte bzw. adaptive Fahrassistenzsysteme Nachhaltigkeitsgewinne im Sinne einer Kraftstoffeinsparung und eines risikoloseren Fahrverhaltens erzielen könnten. Basierend auf dem Drei-Säulen-Modell der Nachhaltigkeit mit den Komponenten Ökologie, Ökonomie und Soziales (Enquete-Kommission 1998) bezieht sich der Beitrag in erster Linie auf die ökologischen und sozialen Aspekte. Der zweite Teil des folgenden Textes besteht aus der Beschreibung der Pilotstudie und deren Zielsetzung sowie aus einigen Schlussfolgerungen, die sich aus den Ergebnissen ziehen lassen.

Es muss bereits an dieser Stelle betont werden, dass es sich allenfalls um Aussagen in der Tendenz handelt, da die empirische Basis der Studie zu gering ist, um verlässlichere Aussagen treffen zu können. Zudem kann man aus methodischer Sicht hinterfragen, inwieweit das induktive statistische Verfahren der Clusterbildung sowie die interpretierende Bewertung der Fahrleistungen belastbare Aussagen zulassen. Ungeachtet dessen halten wir die Schlussfolgerung für richtig, dass die Parametrisierung von Fahrassistenzsystemen anhand von Fahrtypen zur Erhöhung der Akzeptanz in Hinblick auf die beschriebenen Effizienzgewinne kontraproduktiv ist.

\section{(Weiter-)Entwicklung adaptiver Fahr- assistenzsysteme und deren Akzeptanz}

Eine Motivation für die weiter unten vorgestellte Pilotstudie liegt darin, dass heute bereits verbaute Fahrassistenzsysteme nicht immer genutzt werden (Reagan at al. 2018). Aus Sicht der Automobilhersteller stellt diese Verhaltensweise zunächst ein betriebswirtschaftliches und Marketingproblem dar, volkswirt- schaftlich bedeutet es Ressourcenverschwendung und in Hinblick auf Verkehrssicherheit folgt aus der Nichtnutzung von Fahrassistenzsystemen in vielen Fällen, dass das Potenzial für Gewinne im Bereich der Verkehrssicherheit und vielleicht auch der Ressourceneffizienz nicht voll ausgeschöpft wird.

Wie oben bereits angedeutet, stoßen Fahrassistenzsysteme bzw. das teilassistierte ebenso wie das hoch- und vollautomatisierte Fahren immer noch auf erhebliche Skepsis, wobei entsprechende Studien stark länderabhängige und teilweise auch sehr unterschiedliche Ergebnisse zeigen. Das mag u. a. daran liegen, dass im Gegensatz zur etablierten Forschung bzgl. der Akzeptanz technischer Innovationen und technikgestützter Dienstleistungen im Allgemeinen die Forschung zur Kundenwahrnehmung noch in einem frühen Stadium steckt (Woisetschläger 2015). Existierende Studien und Projekte, die nicht zuletzt die individuelle und gesellschaftliche Akzeptanz von Fahrassistenzsystemen in den Blick nehmen, sind z. B.:

- Das seit einigen Jahren laufende Förderprojekt Villa Ladenburg (Fraedrich und Lenz 2014) hat bereits eine Reihe von Publikationen nach sich gezogen, in denen z. B. Entwicklungstrends, Veränderungen der Mobilität durch autonome Fahrzeuge, Geschäftsmodelle und Akzeptanzfragen behandelt werden.

- Arndt (2011) hat ein Modell zur Vorhersage und Analyse der Akzeptanz von Fahrassistenzsystemen entwickelt, um Entscheidungen beim Kauf von Pkw beschreiben und prognostizieren zu können.

- Payre et al. (2014) schlagen in eine ähnliche Kerbe wie Arndt (2011) und untersuchen Einstellungen zu und grundsätzliche Akzeptanz von autonomen Fahrzeugen.

- Verberne et al. (2012) sehen Vertrauen in Fahrassistenzsysteme als wesentlichen Akzeptanzfaktor. Damit FahrerInnen die jeweilige Verkehrssituation besser einschätzen können, obwohl sie nicht (völlig) die Kontrolle über das Fahrzeug besitzen, wird der fahrzeugübergreifende Austausch von Informationen zwischen den Fahrassistenzsystemen vorgeschlagen.

Existierende Fahrassistenzsysteme sind in vielen Ländern bereits wichtiger Bestandteil moderner Fahrzeuge, die FahrerInnen vorrangig bei der Fahrt unterstützen und zeitgleich den Komfort steigern sowie die Unfallgefahr minimieren sollen. Die gerade genannten und vermutlich auch viele andere Studien legen allerdings solche Fahrassistenzsysteme zugrunde, die nicht oder nur in geringem Maße adaptiv und parametrisierbar gestaltet sind. Das Fahrzeugverhalten wird durch die ab Werk vorgegebene Programmierung der Systeme bestimmt und kann von den FahrerInnen kaum oder gar nicht beeinflusst werden.

Um die in den Studien teilweise schlecht ausfallende Akzeptanz und damit den Nutzungsgrad zu steigern sowie ihre Anpassungsfähigkeit an die gegebene Verkehrssituation zu erhöhen wird nun seit einigen Jahren versucht, Fahrassistenzsysteme an die jeweils gegebenen Umweltbedingungen und/oder die Charakteristika der FahrerInnen anzupassen. Beispiele sind 
die Arbeiten von Wang et al. (2014), die Geschwindigkeit und Abstand zum vorausfahrenden Fahrzeug an die individuelle Gasund Bremspedalbedienung anpassen möchten und damit ein erweitertes Adaptive Cruise Control-System (ACC) entwickeln wollen. Dannheim et al. (2013) gehen darüber hinaus und integrieren Wetterinformationen in ein ACC, um ein Intelligent Speed Adaptation-System (ISA) zu realisieren.

Eine Adaption des Fahrstils im Rahmen einer evolutionären Weiterentwicklung existierender Fahrassistenzsysteme könnte auch auf kategorisierten Fahrstilen oder -typen beruhen (Haböck et al. 2016). Die in diesem Bereich durchgeführten Studien befassen sich z. B. mit den Motivationen und Interessens-
Langzeitprofil einer typischen Fahrweise führt. Es wäre so denkbar, dass Fahrassistenzsysteme Fahrstile aus dem tatsächlichen Fahrverhalten lernen, doch dies hätte den Nachteil, dass dabei potenziell auch Fehlverhalten im Hinblick auf die Zielgröße kraftstoffsparendes Fahren gelernt werden könnte. Auf diesen Aspekt wird am Ende des Textes zurückzukommen sein.

Will man Fahrstile zur Auswahl stellen oder das Assistenzsystem an das bisherige Fahrverhalten adaptieren, müssen die Charakteristiken der Fahrstile zunächst vordefiniert werden. Hierzu grundlegende Methoden zu entwickeln und erste Ergebnisse zu erzielen, war Ziel einer Pilotstudie mit explorativem Charakter (Haug et al. 2017), die 2015 und 2016 am Institut für

\section{Sollen Nachhaltigkeitsgewinne erzielt werden, müssten}

\section{Fahrassistenzsysteme ein nachhaltiges Fahrzeugverhalten implementieren statt menschliche Fahrtypen zu kopieren.}

schwerpunkten der FahrerInnen, um Rückschlüsse auf Fahrtypen treffen zu können. So wurden z. B. im Rahmen der Studie „Fernfahrer 2.0: Der Mensch im Transport- und Logistikmarkt" auf Basis von Experteninterviews vier Typen entwickelt: $\operatorname{der} B e-$ rufene, der Überzeugte, der Rationale und der Zufällige (Lohre et al. 2014). Das SANTOS-Projekt (situationsangepasste und Nutzer-Typ-zentrierte Optimierung von Systemen zur Fahrerunterstützung) verfolgt das Ziel, die aktive Sicherheit durch eine situationsangepasste und nutzerorientierte Gestaltung der Assistenz- und Kommunikationsfunktionen im System Fahrer-Fahrzeug-Umgebung zu erhöhen (König et al. 2002). Hier wurden die ProbandInnen, die in einem Demonstrator einen Fahrtest absolvierten, in fünf Fahrtypen eingeteilt: sportlich-draufgängerisch, dynamisch-progressiv, routiniert-abgeklärt, unauffällig-konservativ sowie ängstlich-zurückhaltend.

\section{Pilotstudie}

\section{Zielsetzung}

Eine teilautomatisierte Regelung eines Fahrassistenzsystems kann mit sich bringen, dass FahrerInnen das Gefühl der eigenen Kontrolle verlieren und befürchten, Fahrgewohnheiten aufgeben zu müssen. Hier setzt der Ansatz der Adaption des Fahrassistenzsystems an (Haug et al. 2017). Eine bereits in verschiedenen Fahrzeugen gängige Möglichkeit besteht darin, dass die FahrerIn selbst einen Fahrstil aus einem gegebenen Angebot auswählt. Dies könnte z. B. ein energiesparender Fahrmodus („Eco“) oder ein dynamischer Fahrmodus (,Sportlich“) sein. Vor Beginn einer Fahrt wird dann die FahrerIn automatisch erkannt und die Fahrassistenzsysteme dem entsprechenden Fahrstil passend parametrisiert. Ein adaptives System beruht hingegen auf der kontinuierlichen Beobachtung des Fahrverhaltens, die zu einem
Sozialforschung und Technikfolgenabschätzung (IST) der Ostbayerischen Technischen Hochschule (OTH) Regensburg in $\mathrm{Zu}-$ sammenarbeit mit der Bertrandt $\mathrm{GmbH}$ durchgeführt wurde. Im Rahmen der Studie wurde das Fahrverhalten unterschiedlicher Personengruppen vergleichend untersucht und auf dieser Grundlage Typisierungen vorgenommen.

\section{Forschungsdesign}

Die Methodik stellt eine Kombination und Fortentwicklung bestehender Ansätze dar. Um die Komplexität der Fahrsituation aus verschiedenen Perspektiven erfassen zu können, orientierte sich das Forschungsdesign am Ansatz der Triangulation (Flick 2016), d. h. es beinhaltete mit quantitativen (Fragebogen) wie auch qualitativen Methoden (teilnehmende Beobachtungen, narrative Leitfadeninterviews) mehrere sich ergänzende und gegenseitig korrigierende Methoden. Zur Rekonstruktion der Fahrsituation waren im Testfahrzeug zwei USB-Kameras angebracht, die in Fahrtrichtung filmten sowie die Fahrerin/den Fahrer während der Fahrt aufnahmen. Das Testfahrzeug (Audi A7 mit Automatikgetriebe) war mit diversen computergestützten Messvorrichtungen ausgestattet, welche die technischen Fahrdaten aufzeichneten (Haug et al. 2017). Da für die Studie das Fahrverhalten der ProbandInnen ohne Unterstützung von Fahrassistenzsystemen untersucht werden sollte, wurden vor Beginn der Testfahrten alle integrierten Fahrassistenzsysteme (Abstandsregeltempomat, Spurhalteassistent) deaktiviert.

Damit das Fahrverhalten der ProbandInnen in möglichst vielen unterschiedlichen Szenarien getestet werden konnte, setzte sich die Teststrecke aus den Teilstreckenbereichen Stadtverkehr, Landstraße und Autobahn zusammen. Entsprechend der Angaben des Kartendienstes Google Maps betrug der gesamte Streckenverlauf 55,2 km bei einer durchschnittlichen Fahrtzeit von 1:22 Stunden. Alle teilnehmenden Personen fuhren die gleiche 
Strecke; dabei wurde darauf geachtet, dass sich die Fahrbedingungen möglichst glichen. Die Testfahrten wurden im Herbst durchgeführt.

Die ProbandInnenauswahl erfolgte entsprechend des Ansatzes des Theoretical Samplings mittels einer bewussten Zusammenstellung der Stichprobe durch zuvor festgelegte und für das Forschungsvorhaben relevante Merkmale sowie deren minimale und maximale Kontrastierung. Im Rahmen der Untersuchung unterschiedlicher Fahrstile ergaben sich für den Stichprobenplan folgende Merkmale mit ihren Ausprägungen: Alter (junges Alter [18-25], mittleres Alter [26-55] und gehobenes Alter [ab 56]), Geschlecht (weiblich, männlich) sowie Fahrpraxis (wenig, mittel und viel fahrend), wobei sich die Fahrpraxis aus den jährlich gefahrenen Kilometern und der wöchentlichen Nutzung ergab. Insgesamt wurden 18 ProbandInnen ausgewählt, so dass jede Merkmalskombination vertreten war.

\section{Erhebungsinstrumente}

Die Studie beinhaltete eine Reihe unterschiedlicher Erhebungsinstrumente:

- Die schriftliche Fragebogenerhebung erfolgte kurz vor der Testfahrt. Der standardisierte Fragebogen (Schnell et al. 2013) beinhaltete neben Fragen zu persönlichen soziodemografischen Angaben (Geschlecht, Alter, Dauer des Führerscheinbesitzes, Angaben zum aktuellen persönlichen Empfinden) auch Fragen mit größtenteils 5-stufigen Skalen zur persönlichen Fahrpraxis (durchschnittliche Fahrleistung im Jahr in Kilometer, Angaben zum eigenen bzw. üblicherweise genutzten Pkw, Angaben zur Erfahrung mit Automatikschaltgetrieben und Fahrassistenzsystemen, wöchentliche Fahrtätigkeit, Angaben zur Pkw-Nutzung, zum hauptsächlich gefahrenen Strecken- und Straßentyp) und Fragen zum persönlichen Fahrverhalten (Einstellung zum Autofahren, Angaben zum Verhalten bei Mitfahrern und im allgemeinen Straßenverkehr, angelehnt an König et al. 2002).

- Während der Fahrt wurden folgende Signalmessungen und Kameraaufnahmen automatisiert vorgenommen: Aufnahme der Bussignale (CAN, Flexray), USB-Kamera 1: Aufnahme des Fahrzeuginnenraums, USB-Kamera 2: Aufnahme der vorausliegenden Strecke.

- Die offene teilnehmende Beobachtung während der Fahrt erfolgte durch zwei anwesende BeobachterInnen. Die Anwesenheit von zwei BeobachterInnen (Forscher-Triangulation, vgl. Flick 2016) besitzt den Vorteil, dass einerseits die subjektive Verzerrung der Beobachtung minimiert und andererseits die ProbandInnen durch die/den zweiten BeobachterIn in Gespräche verwickelt und so von der künstlich geschaffenen Beobachtungssituation abgelenkt werden konnten.

- Während der Fahrt wurde ein Beobachtungsprotokoll mit folgendem Inhalt angefertigt: Situationsbeschreibung (Datum, Uhrzeit, Aussagen zum Wetter, Zustand der ProbandInnen vor und während der Fahrt, Anwesende und Besonderheiten), tabellarischer Streckenverlauf (Unterteilung der Teststrecke in kleinere Abschnitte zur besseren Mitschrift der Beobachtungen während der Fahrt), Notizbereich (Platz für gesonderte Notizen) und Einschätzung der FahrerInnenleistung hinsichtlich Sportlichkeit, Verkehrssicherheit und Effizienz in den drei Teilstreckenbereichen Stadt, Land und Autobahn.

- Zusätzlich wurden die Videoaufzeichnungen der Fahrten durch eine/n dritten BeobachterIn gesichtet und ausgewertet.

- Bei dem abschließenden Reflexionsgespräch mit den ProbandInnen über die kurz zuvor erfolgte Testfahrt handelte es sich um eine mündliche teilstrukturierte und leitfadengestützte Befragung.

\section{Auswertung}

Auf Basis des Ratingverfahrens (Pauli 2014) erfolgte im Anschluss an die Fahrt bzw. Sichtung der Videoaufzeichnung eine jeweils durch die drei BeobachterInnen vorgenommene und voneinander unabhängige Bewertung der Fahrleistung der ProbandInnen für die Teilstreckenbereiche Stadt, Land und Autobahn hinsichtlich Verkehrssicherheit, Sportlichkeit und Effizienz. Das Ratingverfahren ist eine Methode, mittels derer die komplexen Merkmale einer Beobachtungssituation bzw. derer jeweiligen Ausprägung eingeschätzt und bewertet werden. Dabei stützt sich das Beobachtungsverfahren auf die Urteilsfähigkeit der BeobachterInnen, welche den Gesamteindruck adäquat widerspiegeln soll (Pauli 2014). Die Fahrleistung der ProbandInnen hinsichtlich Verkehrssicherheit, Sportlichkeit und Effizienz wurde anhand einer 5-stufigen Ratingskala beurteilt, wobei 1 die minimale Ausprägung und 5 die maximale Ausprägung der Fahreigenschaft angibt.

Anhand der Beobachtungsprotokolle wurde jeder Indikator einzeln und unabhängig nach der Methode des Ratingverfahrens von den BeobachterInnen zwischen 1 (minimale Ausprägung des Indikators) und 5 (maximale Ausprägung des Indikators) bewertet.

- Verkehrssicherheit wird durch die Variablen Regelkonformes Verhalten im Straßenverkehr und Aufmerksamkeit sowie die Indikatoren Größe des Abstands (Stadtverkehr, Landstraße, Autobahn), Intensität der Verkehrsbeobachtung, Höhe der Geschwindigkeit (entsprechend StVO), Intensität der vorausschauenden Fahrweise, Intensität der Beachtung der Vorfahrtsregeln, Regelkonformes Verhalten bei der Autobahnauffahrt, Ampelverhalten und Art des Kurvenschneidens beschrieben.

- Sportlichkeit bezeichnet eine sportliche Fahrweise, die sich vor allem in den Variablen Spurführung, Geschwindigkeit und Beschleunigungsverhalten sowie deren Indikatoren Anzahl und Art des Kurvenschneidens, Höhe der Geschwindigkeit auf gerader Strecke (Landstraße und Autobahn), Anzahl und Dauer der Längsbeschleunigung und Kickdowns sowie Anzahl und Art des Bremsverhaltens widerspiegelt.

- Effizienz bezeichnet eine kraftstoffsparende und energieeffiziente Fahrweise, die sich aus den Variablen Geschwindigkeit, Beschleunigung und Vorausschauende Fahrweise mit 
den dazugehörigen Indikatoren Art der Kurvendurchfahrt (Fahrpedal- und Bremstätigkeit), Höhe der Geschwindigkeit auf gerader Strecke (Landstraße und Autobahn), Vergleich zwischen Gas- und Bremsverhalten, Intensität der Längsbeschleunigung sowie Größe des Abstands (Stadtverkehr, Landstraße, Autobahn) ergibt.

Die statistische Auswertung erfolgte mittels Clusteranalyse (Backhaus et al. 2015), einer Methode zur Klassifikation von Personen und ihrer Merkmale. Bei dem verwendeten $k$-Means-Clusterverfahren bildet ein Algorithmus aus den vorgegebenen Daten eine Anzahl an Gruppen, bei denen die Summe der quadrierten Abweichungen von den Cluster-Schwerpunkten minimal ist.

Die Auswertung ergab die drei Fahrtypen Ausgeglichen, Sicher und Effizient sowie Sportlich, die mittels der Variablen der Fremdeinschätzung (Mittelwerte der Indikatoren-Bewertungen für Verkehrssicherheit, Sportlichkeit und Effizienz) und deren Differenzen gebildet wurden. Die Gruppenunterschiede sind signifikant (getestet mittels einfaktorieller ANOVA).

\section{Ergebnis}

- Die Gruppe der Ausgeglichenen kennzeichnet einen annähernd gleich hohen Mittelwert für Sicherheit, Sportlichkeit und Effizienz. ProbandInnen, die diesem Fahrtypus zugeordnet wurden, wiesen eine sowohl verkehrssichere, als auch sportliche und effiziente Fahrweise auf. 10 der 18 Probanden konnten diesem Fahrtypus zugeordnet werden (56\%).

- Die Gruppe Sicher und Effizient ist dadurch gekennzeichnet, dass die Mittelwerte für Sicherheit und Effizienz im Vergleich zu dem Wert für Sportlichkeit höher sind. ProbandInnen, die diesem Fahrtypus zugeordnet wurden, kennzeichnet eine eher verkehrssichere und effiziente als eine sportliche Fahrweise. Diesem Fahrtypus entsprechen 5 von 18 Probanden $(28 \%)$.

- Die ProbandInnen, die dem Fahrtypus Sportlich zugeordnet werden konnten, haben einen höheren Wert bei Sportlichkeit als bei Sicherheit oder Effizienz. 11\% der ProbandInnen wurden diesem Typus zugeordnet.

Bei keinem der drei Fahrtypen konnte ein signifikanter Zusammenhang zu den demografischen Angaben der ProbandInnen (Geschlecht, Alter) oder Angaben zur Fahrpraxis und Fahrleistung festgestellt werden; ein Rückschluss zum Beispiel vom Geschlecht, dem Alter oder der Fahrleistung auf den jeweiligen Fahrstil ist also nicht möglich.

Wie schon bemerkt wurden Sportlichkeit, Sicherheit und Effizienz auf einer Skala von 1 (minimale Ausprägung) bis 5 (maximale Ausprägung) durch die BeobachterInnen bewertet (Fremdeinschätzung). Sowohl im Fragebogen vor der Testfahrt als auch in der Befragung nach der Testfahrt wurden die ProbandInnen gebeten, sich hinsichtlich ihrer Fahrweise selbst einzuschätzen. Zwischen Selbsteinschätzung (Interview) und Fremdeinschätzung konnte kein signifikanter Zusammenhang festge- stellt werden. Gründe hierfür könnten zum einen sein, dass die Selbsteinschätzung nicht valide ist und zum anderen, dass die Definitionen und Vorstellungen einer verkehrssicheren, sportlichen und effizienten Fahrweise zwischen ProbandInnen und BeobachterInnen stark voneinander abweichen.

Der Vergleich der Mittelwerte der Selbst- und Fremdwahrnehmung bzgl. der Einschätzung zur Sicherheit ergibt hierbei, dass sich alle drei Fahrtypen überschätzen, wobei der Fahrtypus Sicher und Effizient sich nur gering überschätzt, der Fahrtypus Ausgeglichen etwas mehr und die Sportlichen am meisten. Bezüglich der Einschätzung der ProbandInnen zur Sportlichkeit im Vergleich mit den Bewertungen der BeobachterInnen schätzt sich der Fahrtypus Ausgeglichen sehr gut ein und unterschätzt sich nur wenig, der Fahrtypus Sicher und Effizient überschätzt sich hingegen und die Sportlichen unterschätzen ihre Fahrleistung hinsichtlich Sportlichkeit sehr. Der Vergleich der Selbstund Fremdwahrnehmung zur Effizienz zeigt, dass der Fahrtypus Sicher und Effizient sich unterschätzt und die Fahrtypen Ausgeglichen und Sportlich sich hinsichtlich ihrer effizienten Fahrweise überschätzen. Mit anderen Worten: Zwei Drittel der ProbandInnen fahren weniger kraftstoffsparend als sie annehmen. Insbesondere dieses Ergebnis muss für die Schlussfolgerungen bzgl. der Unterstützung von Nachhaltigkeit durch Fahrassistenzsysteme beachtet werden.

Wie ein direkter Vergleich der drei Fahrtypen zeigt, weicht der Fahrtypus Sportlich im Vergleich zu den Fahrtypen Sicher und Effizient sowie Ausgeglichen bei allen drei Indikatoren am meisten mit seiner Selbstwahrnehmung von der Fremdeinschätzung ab.

\section{Diskussion}

Da die Untersuchung als eine Art Vorstudie konzipiert war, um mögliche Hinweise auf unterschiedliche Fahrtypen entdecken zu können, ist die Stichprobe sehr klein gehalten. In einer Folgestudie müsste daher die Stichprobe vergrößert werden. Auch ließ sich nicht vermeiden, dass sich die Ausgangsbedingungen bei den Fahrten unterschieden (Wochentag, Uhrzeit, Wetterbedingungen).

Wie die Studie gezeigt hat, kann der Fahrstil anhand der drei Eigenschaften Verkehrssicherheit, Sportlichkeit und Effizienz sowie deren Indikatoren beschrieben werden. Auch wenn die Stichprobengröße sehr gering ist, konnten mittels der Bewertung des Fahrstils der ProbandInnen durch drei BeobachterInnen während der Testfahrten drei signifikant unterschiedliche Fahrtypen definiert werden. Weniger als ein Drittel kann dem ressourcenschonenden Fahrtypus der Sicheren und Effizienten zugeordnet werden.

Weitaus schwerwiegender hingegen scheint, dass zwischen Fremdeinschätzung (Beobachtung) und Selbsteinschätzung kein signifikanter Zusammenhang besteht und Differenzen bei allen Fahrtypen auftraten. Im Durchschnitt unterschätzen sich die Probanden hinsichtlich ihrer sportlichen Fahrweise. In Bezug auf Sicherheit und Effizienz bewerten sich die Befragten jedoch generell selbst besser als die BeobachterInnen; dies gilt in be- 
sonderem Maße für den Fahrtypus der Sportlichen. In Punkto vorausschauende, kraftstoffsparende und energieeffiziente Fahrweise ist Selbstüberschätzung somit weit verbreitet.

\section{Schlussfolgerungen: Adaptiv ja, aber nachhaltig?}

Ob die beschriebene Strategie einer evolutionären Entwicklung von Fahrassistenzsystemen zu einer ökonomisch, ökologisch und sozial nachhaltigen Mobilität beitragen kann, ist mit dieser Pilotstudie nicht endgültig beantwortet, doch lassen die Ergebnisse erhebliche Zweifel zumindest in Bezug auf die ökologische (Kraftstoffverbrauch und in der Folge Schadstoffausstoß) und die soziale (Verkehrssicherheit) Nachhaltigkeitsdimension aufkommen. Betriebswirtschaftlich macht es vermutlich Sinn, Fahrassistenzsysteme schrittweise einzuführen, weil so z. B. Investitionen über längere Zeiträume verteilt und Argumente für neue Fahrzeuggenerationen abgeleitet werden können. $\mathrm{Ob}$ die schrittweise Einführung jedoch volkswirtschaftlich Sinn ergibt und damit die ökonomische Dimension der Nachhaltigkeit adressiert wird, sei dahingestellt - womöglich würden öffentlich geförderte Infrastrukturen aufgebaut, die schon nach eher kurzer Zeit obsolet werden. Das muss hier jedoch eine bloße Vermutung bleiben.

Zieht man nun die oben skizzierten Ergebnisse in Betracht und würden auf Basis entsprechend breit angelegter Studien, die z. B. auf den hier präsentierten Verfahren und Ergebnissen aufbauten, Fahrtypen definiert und programmiert, die die FahrerInnen in Zukunft auswählen könnten, wären die so parametrisierten Fahrassistenzsysteme weniger ressourcensparend und unsicherer als sie durch ihre Benennung signalisierten. Mögliche Effizienzgewinne in Gestalt eines geringeren Kraftstoffverbrauchs oder erhöhter Sicherheit wären mit ihnen also gerade nicht zu erzielen. Dies ist darin begründet, dass die Fahrstile die Fehleinschätzungen der untersuchten FahrerInnen beinhalteten. Denkbar wäre natürlich, dass bei systematischen Verzerrungen diese herausrechenbar wären und so die Fehleinschätzungen nicht in die Parametrisierung der Fahrstile einflössen dann aber spiegelten diese nicht mehr den erwarteten Fahrstil wider und könnten somit auf mangelnde Akzeptanz treffen. Sinn und Zweck parametrisierter Fahrassistenzsysteme in Hinblick auf Akzeptanzsteigerung würden somit verfehlt.

Eine Alternative wäre, Fahrassistenzsysteme nicht ab Werk zu parametrisieren, sondern selbst den Fahrstil der NutzerInnen eines Fahrzeugs erlernen zu lassen, um danach einen der jeweiligen Person angemessenen Fahrstil zeigen zu können. Nach den oben geschilderten Erfahrungen könnte das aber wiederum bedeuten, dass die Fahrzeuge ein Fahrverhalten lernten, das in Hinsicht auf Sicherheit und Kraftstoffverbrauch nicht optimal wäre. Kurzum: Parametrisierte wie adaptive Fahrassistenzsysteme, die dazu dienen sollen, durch eine angepasste assistierte Fahrweise die Attraktivität und Akzeptanz des teilassistierten, hochautomatisierten und irgendwann vollautomatisierten Fahrens zu er- höhen, würden die Argumente, die objektiv für die Nutzung von Fahrassistenzsystemen sprechen, (zumindest teilweise) unterlaufen (vgl. Hartwich et al. 2018).

In Bezug auf die Transformation des bundesdeutschen Verkehrssystems hin zu größerer Nachhaltigkeit ist angesichts dieser Erkenntnisse eher Skepsis angebracht. Sollen Nachhaltigkeitsgewinne - also geringerer Kraftstoffverbrauch und erhöhte Sicherheit - erzielt werden, müssten Fahrassistenzsysteme das Fahrzeugverhalten, der jeweiligen Automatisierungsstufe entsprechend, möglichst vollständig bestimmen und dabei in erster Linie auf ein nachhaltiges Fahrzeugverhalten statt auf die Kopie menschlicher Fahrtypen hin ausgerichtet sein. Das bedeutete notwendigerweise einen weitreichenden Kontrollverlust aufseiten der FahrerInnen. Damit wäre jedoch nicht nur marketingtechnisch eine erhebliche Herausforderung für die Fahrzeughersteller verbunden, sondern dies berührte gerade in Deutschland gesellschaftlich tief verankerte Vorstellungen von Freiheit.

\section{Literatur}

Arndt, Stephanie (2011): Evaluierung der Akzeptanz von Fahrerassistenzsystemen. Modell zum Kaufverhalten von Endkunden. Wiesbaden: vs Verlag für Sozialwissenschaften.

Backhaus, Klaus; Erichson, Bernd; Plinke, Wulff; Weiber, Rolf (2015): Multivariate Analysemethoden. Eine anwendungsorientierte Einführung. Berlin: Springer.

Bertelsmann (2017): Automatisiertes Fahren. Aktuelle Einstellungen in

Deutschland. Gütersloh: Bertelsmann-Stiftung. Online verfügbar unter https://www.bertelsmann-stiftung.de/fileadmin/files/BSt/Publikationen/ GrauePublikationen/Factsheet_LK_Automatisiertes-Fahren_AktuelleEinstellungen-in-Deutschland_2017.pdf, zuletzt geprüft am 08. 05.2018. Dannheim, Clemens; Mader, Markus; Icking, Christian; Loewenau, Jan; Massow, Kay (2013): A novel approach for the enhancement of cooperative ACC by deriving real time weather information. $16^{\text {th }}$ International IEEE Conference on Intelligent Transportation Systems, ITSC 2013. The Hague, New York: IEEE, S. 2207-2211.

Deloitte (2017): What's ahead for fully autonomous driving. Consumer opinions on advanced vehicle technology. Online verfügbar unter https://www2. deloitte.com/content/dam/Deloitte/de/Documents/manufacturing/ us-manufacturing-consumer-opinions-on-advanced-vehicle-technology.pdf, zuletzt geprüft am 08. 05.2018.

Enquete-Kommission (1998): Abschlussbericht der Enquete-Kommission „Schutz des Menschen und der Umwelt - Ziele und Rahmenbedingungen einer nachhaltig zukunftsverträglichen Entwicklung“. Deutscher Bundestag: Drucksache 13/11200. Online verfügbar unter http://dipbt.bundestag.de/ doc/btd/13/112/1311200.pdf, zuletzt geprüft am 08.05.2018.

Ernst \& Young (2017): Autonomes Fahren in Deutschland. Ergebnisse einer Befragung von 1.000 Verbrauchern. Eschborn: Ernst \& Young GmbH. Online verfügbar unter http://www.ey.com/Publication/vwLUAssets/ey-autonomesfahren-in-deutschland/\$FILE/ey-autonomes-fahren-in-deutschland.pdf, zuletzt geprüft am 08. 05.2018.

Flick, Uwe (2016): Qualitative Sozialforschung. Eine Einführung. Hamburg: Rowohlt. Fraedrich, Eva; Lenz, Barbara (2014): Autonomes Fahren. Mobilität und Auto in der Welt von morgen. Ausblick zur Akzeptanz des autonomen Fahrens im Projekt "Villa Ladenburg“ der Daimler und Benz Stiftung. In: TATuP - Zeitschrift für Technikfolgenabschätzung in Theorie und Praxis 23 (1), S. 46-53. 
Haböck, Ulrich; Klier, Janina; Schwenninger, Jochen; Maier, Stefan (2016): Systemadaption als Schlüssel für das automatisierte Fahren. In: Automobiltechnische Zeitschrift (ATZ) 118 (4), S. 26-31.

Hartwich, Franziska; Beggiato, Matthias; Krems, Josef F. (2018): Driving comfort, enjoyment, and acceptance of automated driving. Effects of drivers' age and driving style familiarity. In: Ergonomics.

Haug, Sonja et al. (2017): Identifikation von Fahrtypen. Ein empirischer Ansatz zur Klassifikation von Fahrtypen anhand von Selbst- und Fremdwahrnehmung bei Testfahrten. Begleitstudie zur Entwicklung eines adaptiven Fahrassistenzsystems. Beitrag zur 2. OTH Forschungsclusterkonferenz, OTH Regensburg, 18. 01.2017, Tagungsdokumentation, S. 176-181. Online verfügbar unter https:// www.elektromobilitaet-regensburg.de/uploads/media/Haug_u.a._2017_ Projekt_Fahrtypen_-_Beitrag_OTH_Clustertagung_2017.pdf, zuletzt geprüft am 08. 05.2018

König, Winfried; Weiß, Karl-Ernst; Mayser, Christoph; (2002): S.A.N.T.O.S. Projekt. Situations-angepasste und Nutzer-Typ-zentrierte Optimierung von Systemen zur Fahrerunterstützung. Online verfügbar unter http://edok01.tib.uni-hannover.de/edoks/e01fb02/373006306.pdf, zuletzt geprüft am 08. 05.2018.

Lohre, Dirk; Bernecker, Tobias; Stock, Wilfried, Düsseldorf, Karl (2014): ZFZukunftsstudie Fernfahrer 2.0. Der Mensch im Transport- und Logistikmarkt. Heilbronn: Institut für Nachhaltigkeit in Verkehr und Logistik der Hochschule Heilbronn. Online verfügbar unter http://www.zf-zukunftsstudie.de/ wp-content/uploads/2014/08/ZF-Zukunftsstudie-2014-Gesamt.pdf, zuletzt geprüft am 08.05.2018.

Pauli, Christine (2014): Ratingverfahren. In: Journal für LehrerInnenbildung 6 (1), S. 56-59.

Payre, William; Cestac, Julia; Delhomme, Patricia (2014): Intention to use a fully automated car. Attitudes and a priori acceptability. In: Vehicle Automation and Driver Behaviour 27 (Part B), S. 252-263.

Reagan, Ian; Cicchino, Jessica; Kerfoot, Laura; Weast, Rebecca (2018): Crash avoidance and driver assistance technologies. Are they used? In: Transportation Research Part F: Traffic Psychology and Behaviour 52, S. 176-190. DOI: 10.1016/j.trf.2017.11.015.
Schnell, Rainer; Hill, Paul; Esser, Elke (2013): Methoden der empirischen Sozialforschung. München: Oldenbourg.

Verberne, Frank; Ham, Jaap; Midden, Cees (2012): Trust in smart systems.

Sharing driving goals and giving information to increase trustworthiness and acceptability of smart systems in cars. In: Human Factors 54 (5), S. 799-810.

Wang, Jianqiang; Li, Keqiang; Zhang, Dezhao; Zhang, Lei; (2014): An adaptive longitudinal driving assistance system based on driver characteristics. In: IEEE Transactions on Intelligent Transportation Systems 14 (1), S. 1-12. Woisetschläger, David (2015): Machtauswirkungen des automatisierten Fahrens. In: Markus Maurer, Christian Gerdes, Barbara Lenz und Hermann Winner (Hg.): Autonomes Fahren. Technische, rechtliche und gesellschaftliche Aspekte. Berlin: Springer Vieweg, S. 709-732.

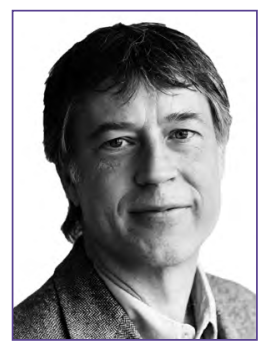

\section{PROF.DR. KARSTEN WEBER}

ist Ko-Leiter des Instituts für Sozialforschung und Technikfolgenabschätzung (IST) der OTH Regensburg sowie Honorarprofessor für Kultur und Technik an der BTU Cottbus-Senftenberg. Er beschäftigt sich derzeit mit individuellen und gesellschaftlichen Auswirkungen von luK-Technologie sowie mit wertebasierter Gestaltung von Technik insbesondere im Gesundheitsbereich.

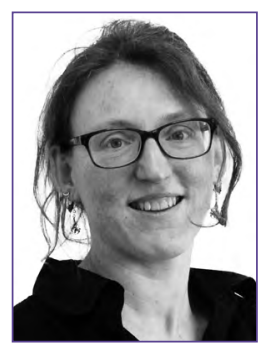

PROF.DR. SONJA HAUG

ist Ko-Leiterin des Instituts für Sozialforschung und Technikfolgenabschätzung (IST) sowie Professorin für Methoden der Sozialforschung und Sozioinformatik der OTH Regensburg. Sie forscht unter anderem zur Technikakzeptanz und -nutzung in den Bereichen Gesundheit, Energie sowie luK-Technologien.

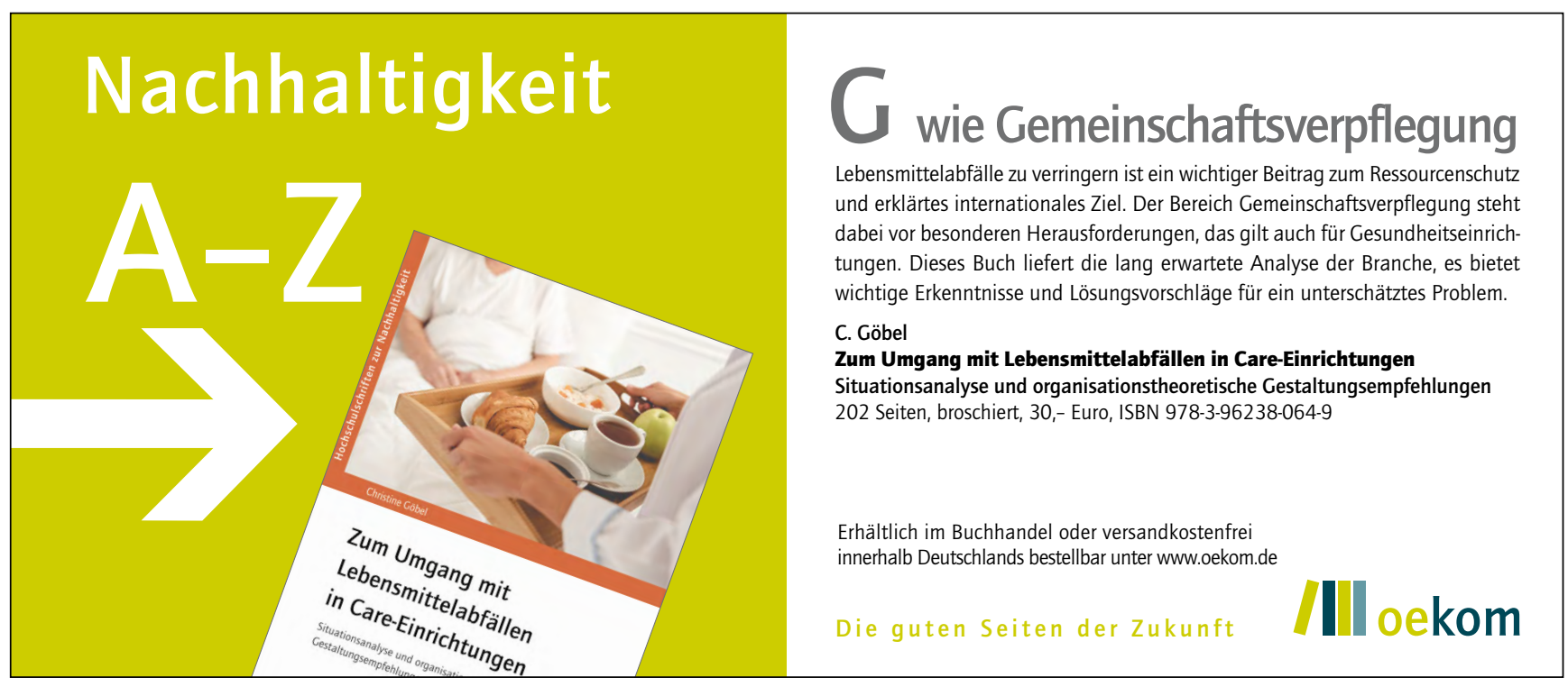

